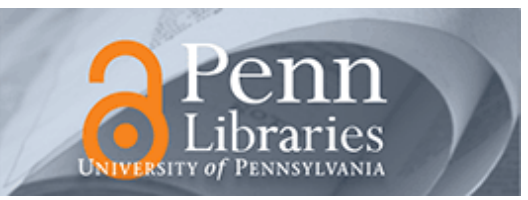

University of Pennsylvania

ScholarlyCommons

\title{
ss-DNA Functionalized Ultra-Thin-Film AIN Contour-Mode Resonators with Self-Sustained Oscillator for Volatile Organic Chemical Detection
}

\author{
Matteo Rinaldi \\ University of Pennsylvania, rinaldim@seas.upenn.edu \\ Brandon Duick \\ University of Pennsylvania, duick@seas.upenn.edu \\ Chiara Zuniga \\ University of Pennsylvania, zunigac@seas.upenn.edu \\ Chengjie Zuo \\ University of Pennsylvania, chengjiezuo@hotmail.com \\ Follow this and additional works at: https://repository.upenn.edu/ese_papers \\ Gianluca Piazza

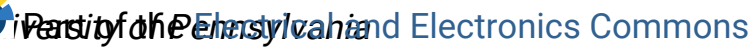

\section{Recommended Citation}

Matteo Rinaldi, Brandon Duick, Chiara Zuniga, Chengjie Zuo, and Gianluca Piazza, "ss-DNA Functionalized Ultra-Thin-Film AIN Contour-Mode Resonators with Self-Sustained Oscillator for Volatile Organic Chemical Detection", 23rd IEEE International Conference on Micro Electro Mechanical Systems (MEMS 2010), 132-135. January 2010.

Suggested Citation:

Matteo Rinaldi, Brandon Duick, Chiara Zuniga, Chengjie Zuo, and Gianluca Piazza. "ss-DNA Functionalized UltraThin-Film AIN Contour-Mode Resonators with Self-Sustained Oscillator for Volatile Organic Chemical Detection" 23rd IEEE International Conference on Micro Electro Mechanical Systems (MEMS 2010) (2010): 132-135.

C2010 IEEE. Personal use of this material is permitted. However, permission to reprint/republish this material for advertising or promotional purposes or for creating new collective works for resale or redistribution to servers or lists, or to reuse any copyrighted component of this work in other works must be obtained from the IEEE."

This paper is posted at ScholarlyCommons. https://repository.upenn.edu/ese_papers/538

For more information, please contact repository@pobox.upenn.edu. 


\title{
ss-DNA Functionalized Ultra-Thin-Film AIN Contour-Mode Resonators with Self- Sustained Oscillator for Volatile Organic Chemical Detection
}

\author{
Abstract \\ This paper reports on the design and experimental verification of a new class of nanoscale gravimetric \\ sensors based on ultra-thin-film AIN Contour-Mode Resonant Sensor (CMR-S) functionalized with ss-DNA \\ and connected to a chip-based self-sustaining oscillator loop (fabricated in the ON Semiconductor $0.5 \mu \mathrm{m}$ \\ CMOS process) for direct frequency read-out. The $220 \mathrm{MHz}$ oscillator based on the ultra-thin AIN CMR-S \\ exhibits an Allan Variance of $\sim 20 \mathrm{~Hz}$ for $100 \mathrm{~ms}$ gate time. The sensor affinity for the adsorption of \\ volatile organic chemicals such as 2,6 dinitroluene (DNT, a simulant for explosive vapors) is enhanced by \\ functionalizing the top gold electrode of the device with a thiol-terminated single stranded DNA sequence \\ (Thiol - 5' CTT CTG TCT TGA TGT TTG TCA AAC 3') enabling the detection of concentrations as low as 1.5 \\ part per billion (ppb).

\section{Disciplines} \\ Electrical and Electronics

\section{Comments} \\ Suggested Citation: \\ Matteo Rinaldi, Brandon Duick, Chiara Zuniga, Chengjie Zuo, and Gianluca Piazza. "ss-DNA Functionalized \\ Ultra-Thin-Film AIN Contour-Mode Resonators with Self-Sustained Oscillator for Volatile Organic Chemical \\ Detection" 23rd IEEE International Conference on Micro Electro Mechanical Systems (MEMS 2010) \\ (2010): 132-135. \\ (C2010 IEEE. Personal use of this material is permitted. However, permission to reprint/republish this \\ material for advertising or promotional purposes or for creating new collective works for resale or \\ redistribution to servers or lists, or to reuse any copyrighted component of this work in other works must \\ be obtained from the IEEE."
}




\title{
SS-DNA FUNCTIONALIZED ULTRA-THIN-FILM ALN CONTOUR- MODE RESONATORS WITH SELF-SUSTAINED OSCILLATOR FOR VOLATILE ORGANIC CHEMICAL DETECTION
}

\author{
M. Rinaldi, B. Duick, C. Zuniga, C. Zuo and G. Piazza \\ University of Pennsylvania, Philadelphia, Pennsylvania, USA
}

\begin{abstract}
This paper reports on the design and experimental verification of a new class of nanoscale gravimetric sensors based on ultra-thin-film AIN Contour-Mode Resonant Sensor (CMR-S) functionalized with ss-DNA and connected to a chipbased self-sustaining oscillator loop (fabricated in the ON Semiconductor $0.5 \mu \mathrm{m}$ CMOS process) for direct frequency read-out. The $220 \mathrm{MHz}$ oscillator based on the ultra-thin AIN CMR-S exhibits an Allan Variance of $\sim 20 \mathrm{~Hz}$ for $100 \mathrm{~ms}$ gate time. The sensor affinity for the adsorption of volatile organic chemicals such as 2,6 dinitroluene (DNT, a simulant for explosive vapors) is enhanced by functionalizing the top gold electrode of the device with a thiol-terminated single stranded DNA sequence (Thiol - 5' CTT CTG TCT TGA TGT TTG TCA AAC 3') enabling the detection of concentrations as low as 1.5 part per billion (ppb).
\end{abstract}

\section{INTRODUCTION}

In recent years the use of micro and nano electromechanical (MEMS/NEMS) resonators for sensing applications has been extensively explored. The scaling of the device dimensions enables the fabrication of ultra-sensitive nanomechanical gravimetric sensors that thanks to their small form factor and IC integration capability can be arranged in single-chip multi-frequency sensor arrays capable of selectively detecting extremely small concentrations (ppb) of multiple gaseous analytes. Such smart, compact, portable and low cost electronic noses for multiple gas detection cannot be implemented with bulky and unintegrable Quartz Crystal Microbalances (QCMs). Even if QCMs have been successfully employed as gravimetric sensors thanks to their extremely high quality factors, which permit to obtain limits of mass detection in the order of few nanograms, their relatively large volume and their inability to be directly integrated on silicon render them unattractive for the fabrication of sensor arrays composed of a large number of sensing elements.

In response to this growing demand for miniaturization, different MEMS/NEMS resonator technologies based on electrostatic or piezoelectric transduction [1,2] have been proposed. In particular, NEMS resonators have been exploited as transducers suitable for the realization of extremely sensitive gravimetric sensors. Sub-attogram mass resolution has been demonstrated in NEMS cantilevers [3] thanks to the minuscule mass and the relatively high quality factor $(Q)$. Nevertheless the greatly reduced dimensions of these devices render their transduction extremely hard to implement and requires the use of cumbersome, complex and power inefficient read-out techniques.

These issues have been recently overcome by AlN Contour-Mode Resonant Sensors (CMR-S) [4, 5, 6]. In fact, this technology enables the fabrication of ultra-sensitive nanoscaled devices that can be piezoelectrically actuated and sensed directly on chip [5]. In addition, differently from conventional resonant sensors, such as QCMs or FBARs, whose operating frequency (set by the thickness of the piezoelectric layer) is intrinsically related to the sensitivity of the device to mass loading, the AlN CMR-S uses the film thickness to set the device sensitivity independently from the frequency of operation.

In this work the thickness of the AIN CMR-S has been scaled to the nano range ( $250 \mathrm{~nm}$ thick) in order to achieve high values of sensitivity to mass loading ( $\geq 40 \mathrm{kHz} \cdot \mu \mathrm{m}^{2} / \mathrm{fg}$ ). Furthermore, thanks to the large value of the resonator figure of merit $\left(k_{t}^{2} \cdot Q\right.$, product of the electromechanical coupling and resonator $Q$ ) in excess of 20, direct wire-bonding of the CMR-S to a

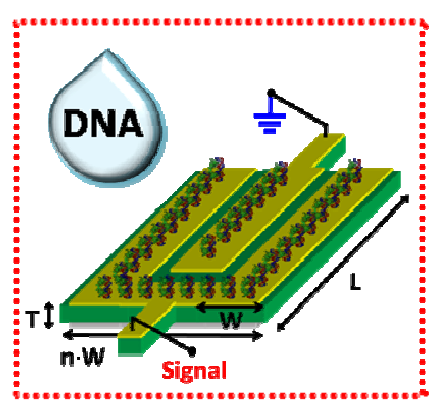

Ultra-Thin Film AIN CMR-S

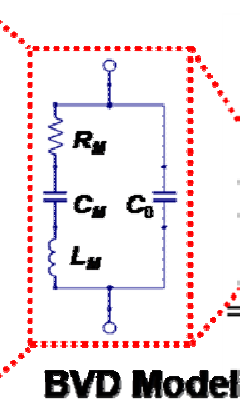

BVO Model

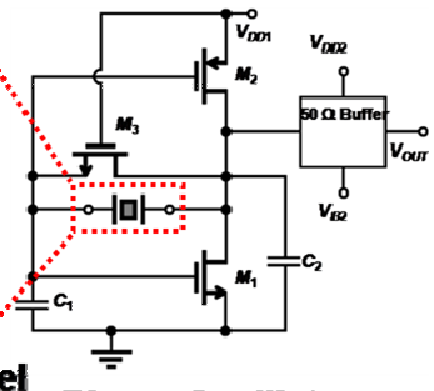

Pierce Oscillator
Figure 1: $\quad$ Schematic representation of the fabricated nanoenabled ultrathin film AlN CMR-S connected in a self-sustained oscillator loop (Pierce oscillator). The butterworth van dyke (BVD) model is used to describe the resonator behavior. 
Pierce oscillator [7] fabricated in the ON Semiconductor $0.5 \mu \mathrm{m}$ CMOS process was made possible. The sensor affinity for the adsorption of volatile organic chemicals such as 2,6 dinitroluene was enhanced by functionalizing the top gold electrode of the device with a thiol-terminated single stranded DNA sequence (Thiol - 5' CTT CTG TCT TGA TGT TTG TCA AAC 3') and the ability to detect concentrations as low as $1.5 \mathrm{ppb}$ was demonstrated.

\section{NANOSCALED CMR-S DESIGN}

A CMR is composed of an AlN film sandwiched between two patterned metal electrodes (Fig. 1). When an AC votlage is applied across the thickness ( $T$ ) of the device a contour-extensional mode of vibration is excited thorough the equivalent $d_{31}$ piezoelectric coefficient of AlN. Given the equivalent mass density, $\rho_{e q}$, and Young's modulus, $E_{e q}$, of the material stack (AIN and metal electrodes) that forms the resonator, the center frequency, $f_{0}$, of this laterally vibrating mechanical structure, is univocally set by the period, $W$, of the metal electrode patterned on the AIN plate and can be approximately expressed as in Eq. (1).

$$
f_{0}=\frac{1}{2 W} \sqrt{\frac{E_{e q}}{\rho_{e q}}}
$$

The other two geometrical dimensions, thickness, $T$, and length, $L$, set the equivalent electrical impedance of the resonator [2] and can be designed independently of the desired resonance frequency.

The sensitivity to mass per unit area of a CMR-S loaded on its top surface can be expressed as in Eq. (2) $[5,6]$.

$$
S_{C M R-S}=-\frac{f_{0}}{2 \rho_{e q} T}
$$

It is worth nothing that the resonance frequency, $f_{0}$, and the thickness, $T$, of the CMR-S are two independent variables while for FBARs and QCMs they are intrinsically coupled and cannot be set independently from one another. This is an important and unique advantage of CMR-S. In fact, it permits to set the frequency of operation of these devices according to the specifications of the desired application and independently obtain the required value of mass sensitivity by scaling the device thickness.

Nevertheless, the mass sensitivity of the device cannot be considered the only important parameter for the design of a high performance gravimetric sensor. In fact, the limit of detection, $L O D$, of the sensor (smallest amount of adsorbed mass per unit area that can be resolved) is defined as in Eq. (3):

$$
L O D=\frac{\Delta f_{\min }}{S_{C M R-S}}
$$

where $\Delta f_{\min }$ indicates the minimum frequency shift detectable by the sensor read-out. Thus, the performance of the sensor can be improved by reducing the thickness, $T$, of the AIN layer up to the limit at which the smallest value of the ratio, $\Delta f_{\min } / f_{0}$, is preserved. For example, $\Delta f_{\min } / f_{0}$ comparable to thicker films and as low as $9 \cdot 10^{-8}$ has been attained for the ultra-thin device of this work.

Another crucial aspect directly related to the scaling of the device is the complexity, size and power efficiency of the required sensor read-out. In particular, the ability to connect the nanoelectromechanical resonator to a self-sustained oscillator loop for direct frequency read-out greatly simplifies the design of multiplexed sensor platforms involving large arrays of resonant devices. Selfsustained, high performance, Pierce-like oscillator circuits based on thick film $(2-3 \mu \mathrm{m})$ AlN Contour_Mode MEMS Resonator (CMR) have been previously demonstrated by our group [7]. The primary power loss in these oscillator circuits is due to the motional resistance, $R_{m}$, of the resonator [7], whose value is inversely proportional to the device figure of merit, $k_{t}^{2} \cdot Q$ [8]. Therefore, the $k_{t}^{2} \cdot Q$ product of the nanoscaled CMR needs to be maintained sufficiently large $(>10)$ to be able to connect it to a compact and low power self-sustained oscillator circuit for direct frequency read-out.

According to these considerations the frequency of operation of the CMR-S presented in this work was set to $220 \mathrm{MHz}(W=15 \mu \mathrm{m})$ and the thickness of the AlN layer was scaled in the nano domain $(T=$ $250 \mathrm{~nm}$ ) in order to improve the sensitivity of the resonant sensor. Differently from conventional CMRs, a scheme based on lateral field excitation (LFE) [5] is adopted to excite a higher order contourextensional mode of vibration in the nanoscaled AlN plate. The very thin $(250 \mathrm{~nm})$ AlN film (composing the body of the resonator) is deposited on top of a floating bottom Platinum $(\mathrm{Pt})$ electrode which acts to confine the electric field across the thickness of the device and provides for a high $k_{t}^{2}(1.6 \%$ in this work).

The oscillator circuit topology used as read-out for the CMR-S of this work is described in [7]. Briefly, the circuit (Fig. 1) consists of a Pierce oscillator implemented by means of a CMOS inverter biased in its active region. Transistors M1 and M2 form the CMOS inverter while transistor M3 acts as a large resistor to provide biasing in the active region of M1 and M2.

In order to enable the functionalization of the sensor with thiolated single stranded DNA (HSssDNA) sequences, the top metal electrode of the CMR-S was made out of gold [9]. The capability of single stranded DNA to selectively enhance the adsorption of volatile organic compounds such as dymethyl-methylphosphonate (DMMP, a simulant for nerve agent sarin) and 2,6 dinitroluene (DNT, a simulant for explosive vapors) has been recently demonstrated by our group. Single wall carbon 
nanotube (SWNTs) directly grown on the top surface of a CMR-S [4] were functionalized by the DNA strands. In this work, in order to enhance the sensor affinity for the adsorption of volatile organic chemicals, the top gold electrode of the device was directly functionalized (without any SWNT interface layer) with a thiol-terminated ss-DNA sequence.

\section{EXPERIMENTAL RESULTS}

A 4 mask fabrication process, analogue to the one reported in [6], was employed to fabricate the devices presented in this work.

The electrical response of the fabricated ultrathin-film AIN CMR-S was characterized in ambient conditions in an RF probe station and the admittance curve measured by an Agilent ${ }^{\circledR}$ N5230A Network Analyzer after performing a short-open-load (SOL) calibration on a reference substrate.

The electrical response of the device was fitted to the Butterworth van Dyke (BVD) equivalent circuit (Fig. 1). Both the experimental and fitted admittance curves of the device are shown in Figure 2.

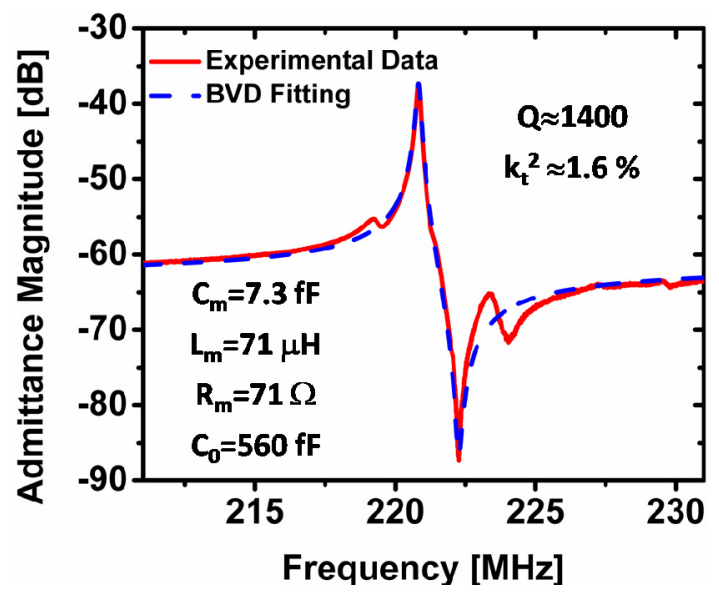

Figure 2: Experimental and fitted (BVD model) admittance curves of the fabricated ultra-thinfilm AlN CMR-S.

The sensitivity to mass loading of the fabricated device was extracted to be approximately $41.6 \mathrm{KHz} \cdot \mu \mathrm{m}^{2} / \mathrm{fg}$. Despite the device volume reduction, high $\mathrm{Q}(\sim 1,400$ in air $)$ and high $\mathrm{k}_{\mathrm{t}}^{2}(\sim 1.6$ $\%)$ were attained (Fig. 2) enabling the direct wirebonding of the nanoscaled CMR-S to the oscillator circuit chip fabricated in the ON Semiconductor 0.5 $\mu \mathrm{m}$ CMOS process. The $220 \mathrm{MHz}$ oscillator uses a $1.67 \mathrm{~V}$ supply voltage and $300 \mu \mathrm{A}$ of biasing current, which give a total power consumption of $501 \mu \mathrm{W}$. Both the resonator die and the CMOS chip were attached to a custom designed PCB (Fig. 3) which served as the lid for the testing chamber [6].

The sensor was exposed to DNT vapor (generated by bubbling method [6]) and the frequency shift due to the adsorption of the analyte on

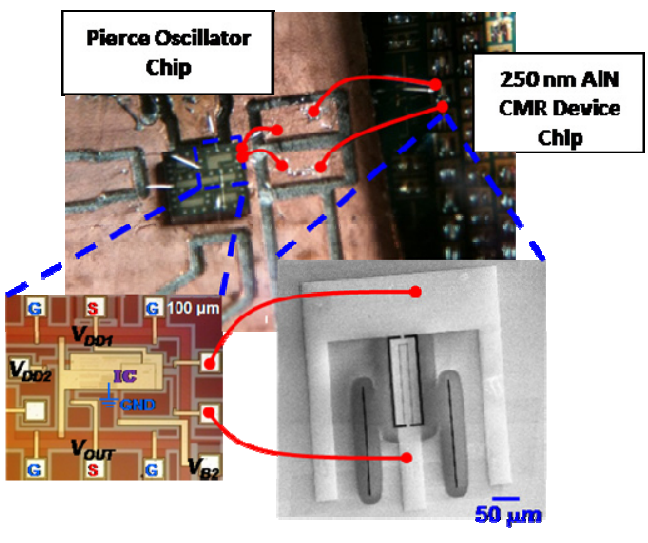

Figure 3: Picture of the ultra-thin film AlN CMR$S$ wire-bonded to the Pierce oscillator chip fabricated in the ON Semiconductor $0.5 \mu \mathrm{m}$ CMOS process.

the $\mathrm{Au}$ electrode (without any functionalization layer) was first monitored with an an Agilent ${ }^{\circledR}$ 8562EC spectrum analyzer (Fig. 4).

The top gold electrode of the ultra-thin film CMR-S was then functionalized with the thiolterminated DNA sequence (obtained from Invitrogen) by micropipetting on the resonator a $2 \mu \mathrm{L}$ drop of $100 \mu \mathrm{M}$ DNA in $1 \mathrm{M}$ Potassium Phosphate monobasic buffer solution [9]. The drop was let rest for 45 minutes in a humid environment and finally dried in a nitrogen stream. After functionalization, the output frequency of the oscillator was lowered by $1 \mathrm{MHz}$, but no significant changes in the oscillator signal were observed. The DNA-functionalized sensor was exposed again to the same 5\% concentration of DNT vapor showing about 8 fold enhancement (Fig. 4) in the binding affinity between the analyte and the resonator surface (amount of adsorbed mass for a given concentration).

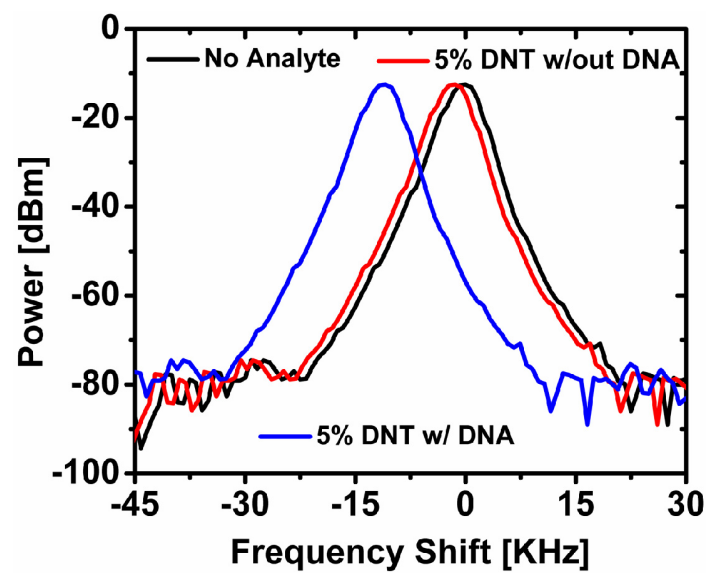

Figure 4: Sensor response to 5\% DNT vapor pressure before and after DNA functionalization.

The improved affinity of the CMR-S to the DNT vapor enabled the detection of smaller analyte concentrations. The measured sensor response for 
different concentrations of DNT, ranging between $0.2 \%$ and $1 \%$ of the saturated vapor pressure, are reported in Fig. 5. Considering that the DNT saturated vapor pressure at room temperature is equal to $5.67 \cdot 10^{-4}$ Torr [10], a concentration of DNT as low as $1.5 \mathrm{ppb}$ was detected by the fabricated nanoenabled CMR-S prototype.

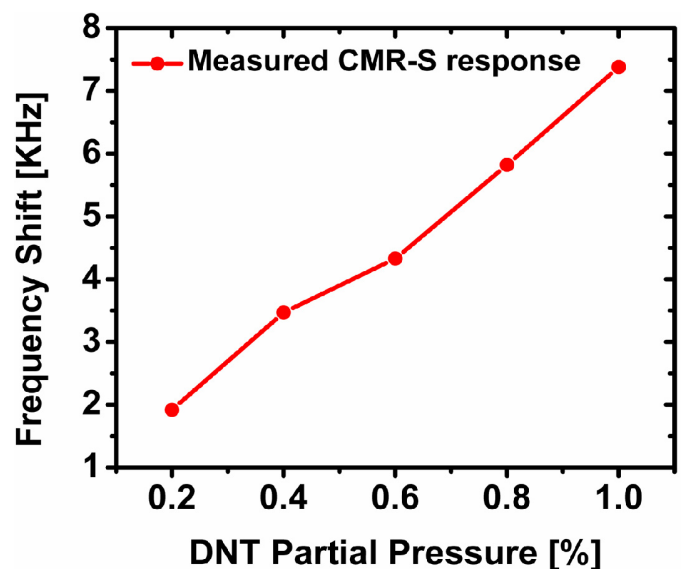

Figure 5: Response of the nanoenabled CMR-S for different concentrations of DNT ranging between $0.2 \%$ and $1 \%$ of the saturated vapor pressure.

In order to estimate the noise limited minimum frequency shift detectable by the sensor read-out $\left(\Delta f_{\min }\right)$, the short term frequency stability of the oscillator output signal was characterized by measuring its Allan variance, $\sigma_{Y}(\tau),[11]$. The frequency measurements were taken with an Agilent ${ }^{\circledR}$ 53132A Universal Frequency Counter and the Allan variance was calculated, for different values of averaging time interval (gate time), $\tau$, according to Eq. (4).

$$
\sigma_{Y}(\tau)=\frac{1}{2(N-1)} \sum_{m=2}^{N}\left(f_{m}-f_{m-1}\right)^{2}
$$

where $f_{m}$ is the average frequency measured over the $m$ th time interval of length, $\tau$, and $N$ is the number of frequency measurements, set to be equal to 100 . The measured values of Allan variance are reported in Fig. 6. The minimum value of Allan variance recorded is approximately $20 \mathrm{~Hz}$ and it is achieved for a gate time, $\tau$, of $100 \mathrm{~ms}$. Therefore, considering a minimum detectable frequency shift, $\Delta f_{\min }$, of $20 \mathrm{~Hz}$, a limit of detection of mass per unit area of about $481 \mathrm{zg} / \mu^{2}$ can be estimated. This translates in an extrapolated limit of detection for DNT concentrations of about 15 part per trillion (ppt).

\section{CONCLUSION}

In this paper, the design and the experimental verification of a new class of nanoscale gravimetric sensors based on ultra-thin film AlN CMR-S functionalized with ss-DNA and connected to a chip- based self-sustaining oscillator loop for direct frequency read-out was demonstrated. Thanks to the large value of the resonator figure of merit, $k_{t}^{2} \cdot Q$, direct wire-bonding of the CMR-S to a Pierce oscillator fabricated in the ON Semiconductor $0.5 \mu \mathrm{m}$ CMOS process was made possible.

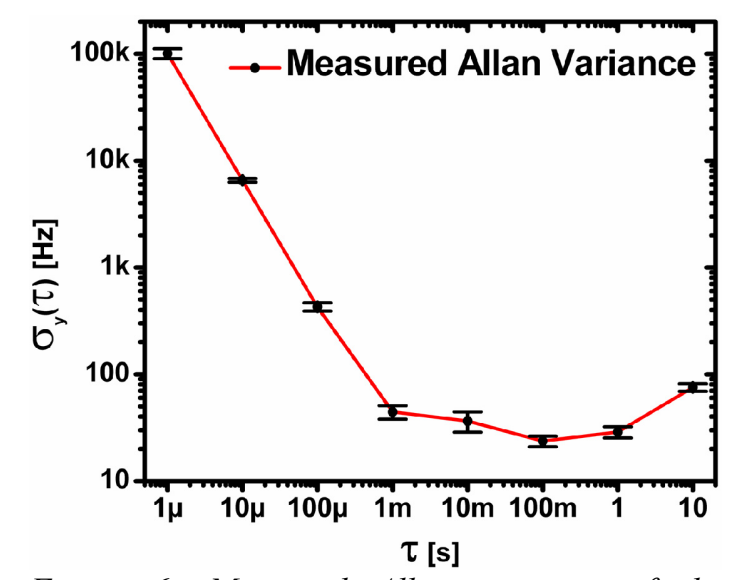

Figure 6: Measured Allan variance of the $220 \mathrm{MHz}$ oscillator.

The power consumption of the sensor prototype was limited to approximately $500 \mu \mathrm{W}$ despite the use of an old CMOS technology. The sensor affinity for the adsorption of volatile organic chemicals such as DNT has been improved by functionalizing the top gold electrode of the device with a thiol-terminated single stranded DNA sequence. Concentrations of DNT as low as $1.5 \mathrm{ppb}$ were measured. Thanks to the high frequency stability of the oscillator signal (minimum Allan variance of $20 \mathrm{~Hz}$ for $100 \mathrm{~ms}$ gate time), a limit of detection of $15 \mathrm{ppt}$ of DNT can be extracted for the fabricated CMR-S prototype.

\section{REFERENCES}

[1] K. Wang, A.-C. Wong and C. T.-C. Nguyen, J. Microelectromech. Syst., vol. 9, no. 3, pp. 347-360, 2000.

[2] G. Piazza, P.J. Stephanou, A.P. Pisano, J. Microelectromech. Syst., vol. 15, no.6, pp. 1406-1418, 2006.

[3] M. Li, H. X. Tang, M.L. Roukes, Nature Nanotechnology, vol. 2, pp. 114-120, 2007.

[4] C. Zuniga, M. Rinaldi, S. M. Khamis, A. T. Johnson, and G. Piazza, Applied Physics Letters, vol. 94, 223122, 2009.

[5] M. Rinaldi, C. Zuniga, and G. Piazza, Proc. $22^{\text {nd }}$ IEEE International Conference on Micro Electro Mechanical Systems (MEMS 2009), Sorrento, Italy, Jan. 2009, pp. 916919.

[6] M. Rinaldi, C. Zuniga, and G. Piazza, Poc. IEEE International Ultrasonics Symposium, Rome, Italy, Sept. 2009 , in press.

[7] C. Zuo, J. Van der Spiegel, and G. Piazza. Proceedings IEEE International Frequency Control Symposium (EFTF-IFCS 2009), Besancon, France, April 2009, pp. 381-384.

[8] K.M. Lakin, Proc. IEEE MTT-S Int. Microwave Symp. Dig., June 1992, pp. 149-152.

[9] T. M. Herne and M. J. Tarlov, J. Am. Chem. Soc., 119, pp. 8916-8920, 1997.

[10] Regulatory Determinations Support Document for Selected Contaminants from the Second Drinking Water Contaminant Candidate List (CCL 2), United States Environmental Protection Agency, Chapter 7, June 2008.

[11] D. W. Allan, Proc. IEEE, 54, 2, pp. 221-230, 1966. 